Article

\title{
Estimating Human Body Dimensions Using RBF Artificial Neural Networks Technology and Its Application in Activewear Pattern Making
}

\author{
Zhujun Wang ${ }^{1,2,3,4}$, Jianping Wang ${ }^{1,5, *}$, Yingmei Xing ${ }^{2,3,4}$, Yalan Yang ${ }^{1}$ and Kaixuan Liu ${ }^{6}$ \\ 1 College of Fashion and Design, Donghua University, Shanghai 200051, China; \\ hqxiaopan@126.com (Z.W.); Yangyalan821@126.com (Y.Y.) \\ 2 School of Textile and Garment, Anhui Polytechnic University, Wuhu 241000, China; yingmei82@126.com \\ 3 Anhui Province College Key Laboratory of Textile Fabrics, Anhui Polytechnic University, \\ Wuhu 241000, China \\ 4 Anhui Engineering and Technology Research Center of Textile, Anhui Polytechnic University, \\ Wuhu 241000, China \\ 5 Shanghai Institute of Design and Innovation, Tongji University, Shanghai 200092, China \\ 6 Apparel and Art Design College, Xi'an Polytechnic University, Xi'an 710048, China; L40260611@hotmail.com \\ * Correspondence: wangjp@dhu.edu.cn
}

Received: 22 December 2018; Accepted: 13 March 2019; Published: 18 March 2019

\begin{abstract}
Nowadays, the popularity of the internet has continuously increased. Predicting human body dimensions intelligently would be beneficial to improve the precision and efficiency of pattern making for enterprises in the apparel industry. In this study, a new predictive model for estimating body dimensions related to garment pattern making is put forward based on radial basis function (RBF) artificial neural networks (ANNs). The model presented in this study was trained and tested using the anthropometric data of 200 adult males between the ages 20 and 48 . The detailed body dimensions related to pattern making could be obtained by inputting four easy-to-measure key dimensions into the RBF ANN model. From the simulation results, when spreading parameter $\sigma$ and momentum factor $\alpha$ were set to 0.012 and 1, the three-layer model with 4,72 , and 8 neurons in the input, hidden, and output layers, respectively, showed maximum accuracy, after being trained by a dataset with 180 samples. Moreover, compared with a classic linear regression model and the back propagation (BP) ANN model according to mean squared error, the predictive performance of the RBF ANN model put forward in this study was better than the other two models. Therefore, it is feasible for the presented predictive model to design garment patterns, especially for tight-fitting garment patterns like activewear. The estimating accuracy of the proposed model would be further improved if trained by more appropriate datasets in the future.
\end{abstract}

Keywords: human body dimension; tight-fitting garment pattern making; activewear; radial basis function; artificial neural networks

\section{Introduction}

In recent years, requirements for individualized garments have increased rapidly, including clothing styles, colors, and fabrics. However, excellent garment fit is indispensable, which is considered to be a critical factor affecting garment wearing comfort. In today's apparel industry, garment pattern making is a vital procedure of manufacturing well-fitting garments. Garment patterns, also known as paper patterns, refer to paper or cardboard templates based on which the parts of the garment are draw on the fabric before cutting out. Making garment patterns, sometimes called garment structure design, pattern design, pattern drafting, or pattern cutting, is a complicated technique, involving a 
wide range of knowledge (e.g., aesthetics, mathematics, ergonomics). The key problem of making well-fitting garment patterns is to design garment sizes or dimensions, heavily depending on the expertise and experiences of pattern makers. Generally, the garment sizes are designed and adjusted by pattern makers according to human body dimensions. Individualized garments need more accurate human body dimensions. Therefore, anthropometric measurement is an essential prerequisite for pattern making.

Currently, shopping over the internet has become more incorporated into people's lifestyle with the continuously increasing popularity of the internet. Garment suppliers have been challenged in providing individualized garments that fit exactly a certain consumer's body size and body shape, due to the obstacle of procuring human body dimensions directly and precisely through the internet. Ordinarily, human body dimensions could be measured manually or automatically. With the advantages of intuitionistic and convenient tools, manual measurement with tapes has been applied as a conventional method of human body data acquisition for years. However, since the method is greatly dependent on the experience and judgement of the measurers, the precision of the data procured is unreliable, which may easily lead to the problem of poor garment fit. Additionally, it is also time-consuming. Compared with the manual anthropometric measurement, the efficiency and accuracy has been greatly improved by 3D human body scanning technology. Over the past decade, 3D human body scanners of various kinds have emerged on the market and been employed in the apparel industry, such as laser scanning, patterned light projection, stereo photogrammetry, millimeter waves, and infrared waves [1,2]. Contributing to the development of CCD-chips (Charge-coupled Device), the 3D body scanners have the advantages of high resolution (1-8 $\mathrm{mm}$ ) and speed $(0.2-3 \mathrm{~s})$, which makes it possible to collect the whole body's data precisely and economically [1,2]. However, since the subjects are required to be naked or wear underwear during the process of body scanning, numerous consumers refuse to be measured. Some other disadvantages of the devices, including the bulky design, high price and huge storage capacity for 3D images, have also influenced their further application in the garment industry, particularly in middle and small-scale garment enterprises [2,3]. Obviously, in the context of sales over the internet, it is unrealistic to utilize the two anthropometric measurement manners aforementioned [4]. In addition, garment enterprises should meet the personalized needs for consumers as soon as possible. Therefore, key dimensions (e.g., body height, bust circumference, waist circumference) which are easy-to-measure are measured physically, while the other detailed dimensions are calculated by inputting the key dimensions into empirical formulas based on linear regression (LR) models. For example, the sleeve length could be calculated by inputting human body height into an LR model. Contributing to inherent simplicity, LR models have been widely applied in the fields of industrial product design including garments, tools, furniture, and workplace [5-9]. However, these models are not accurate enough [9]. Thus, it is necessary to develop an approach of obtaining body dimensions used for garment pattern making faster and more accurate than the current methods.

With the rapid development of artificial intelligence (AI) nowadays, predicting human body dimensions by AI rather than measuring them physically has attracted more and more attention in the apparel industry. Recently, due to the advantages of artificial neural networks (ANNs), such as good non-linear approximation abilities and adaptive and self-organizing abilities, and as one of the most popular machine learning approaches, ANN technology has been widely in many fields, including viscosity prediction of nanofluid, human behavior prediction, pattern recognition, and adaptive control [10-14]. In the field of garment pattern making, Chan et al. [15] presented an artificial neural network model to predict the pattern parameters of men's shirt in 2003. However, the inputs of the model proposed consisted of 58 body dimensions which were rather complicated and not easy to collect simultaneously. In 2014, one study by Zheng Liu et al. [16] put forward a non-linear model to predict the detailed body sizes using feature parameters extracted by principle component analysis. But the feature parameters used as the inputs of the proposed model were difficult to calculate in the research. Another study by Kaixuan Liu et al. [17] in 2017 developed a back propagation (BP) neural networks 
model to predict lower body dimension used for pants pattern design. However, the application of other neural networks models was not mentioned in that study. What is more, little attention has been focused on the application of radial basis function (RBF) ANN in garment pattern making. Therefore, the aim of this paper is to put forward a new ANN model based on radial basis function to improve the precision of estimating body dimensions used for garment pattern making. Additionally, the proposed model could be used for pattern makers with a lack of expertise and experience, through inputting the learning data based on the knowledge of experienced pattern makers.

The rest of the sections of this study are organized as follows. The "Methodology" section expounds on the research scheme and procedures in this paper, including anthropometric data acquisition, and construction of the RBF ANN-based predictive model. In the "Results and Discussion" section, key factors affecting predictive precision of the proposed model are analyzed and an application of the model is put forward and in making the patterns of active leggings. Finally, the "Conclusion" is presented with the conclusions and possible future works.

\section{Methodology}

\subsection{Research Scheme}

The proposed approach for estimating body dimensions used for garment pattern making is described in Figure 1. The detailed implementation process is as follows. First, the anthropometric data of a group of 200 males were gathered to construct the human body dimensions database after data preprocessing. In the sequential step, the data in the database were divided into two groups: the key dimensions and the difficult-to-measure detailed dimensions. Then, the ANN predictive models with different mathematical algorithms were designed, which used the key dimensions as the input variables and the detailed dimensions needed for garment pattern making as the output variables. Afterwards, the constructed models were trained and tested by the training dataset and the testing dataset selected from the database, respectively. Subsequently, the predictive performance of the RBF ANN model proposed was compared with the BP ANN model and the linear regression model.

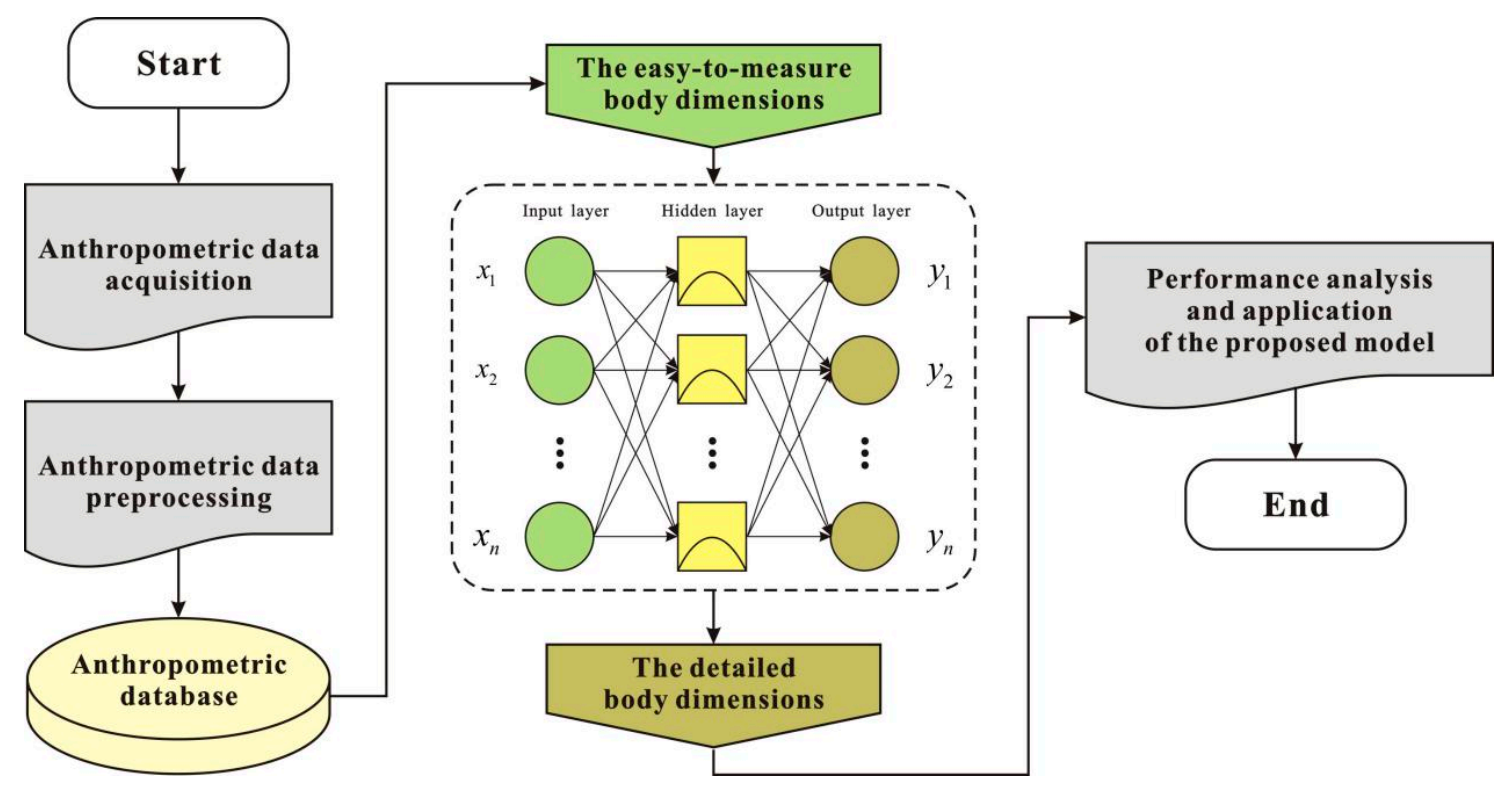

Figure 1. Flow chart.

As a popular type of clothing, tight-fitting activewear without any ease allowance was taken as the study sample, in order to verify the performance of the presented model further. The key body dimensions of a new subject were inputted into the model. Then, the body dimensions related to making the activewear patterns were estimated by the model. Since the activewear selected was 
without any ease allowance, the body dimensions outputted could be utilized as the pattern dimensions. The activewear patterns were made ultimately based on the estimated body dimensions.

\subsection{Anthropometric Data Acquisition and Preprocessing}

With the advantages of taking automatic body measurements precisely within a few seconds, the VITUS 3D body scanner was employed to collect the anthropometric data of 200 adult males who were from the middle and south region of China. The ages of the subjects in this study were between 20 and 48 years of age, and height ranged from 150 to $183 \mathrm{~cm}$.

In the following stage, the data obtained from the 3D scanner were preprocessed by exploratory analysis. The data preprocessing aimed to figure out the singular values influencing the analysis results and to investigate the data samples' distribution. The singular values were mainly induced by measurement errors and special body types. For the values induced by measurement errors, the data were re-measured carefully. For the values induced by special body types, all the measurements were reserved for the following study.

As an increasingly popular style of clothing, active leggings were taken for instance in this study. Since active leggings are tight fitting, their pattern dimensions are strongly related to human body dimensions. Twelve lower-body dimensions related to garment making patterns of active leggings were selected from the database for further study. Figure 2 illustrates the anthropometric dimensions used in this study, including stature, waist height, abdomen height, hip height, crotch height, knee height, waist girth, abdomen girth, hip girth, crotch length, thigh girth, and knee girth. How the dimensions were measured is explained in Table 1. The subject stood upright and without shoes as shown in Figure 2 when being measured by the 3D body scanner. Head line was defined as the top of the head, and the heel line referred to the soles of the feet. The selected anthropometric data were conducted by descriptive analysis and the results are given in Table 2.

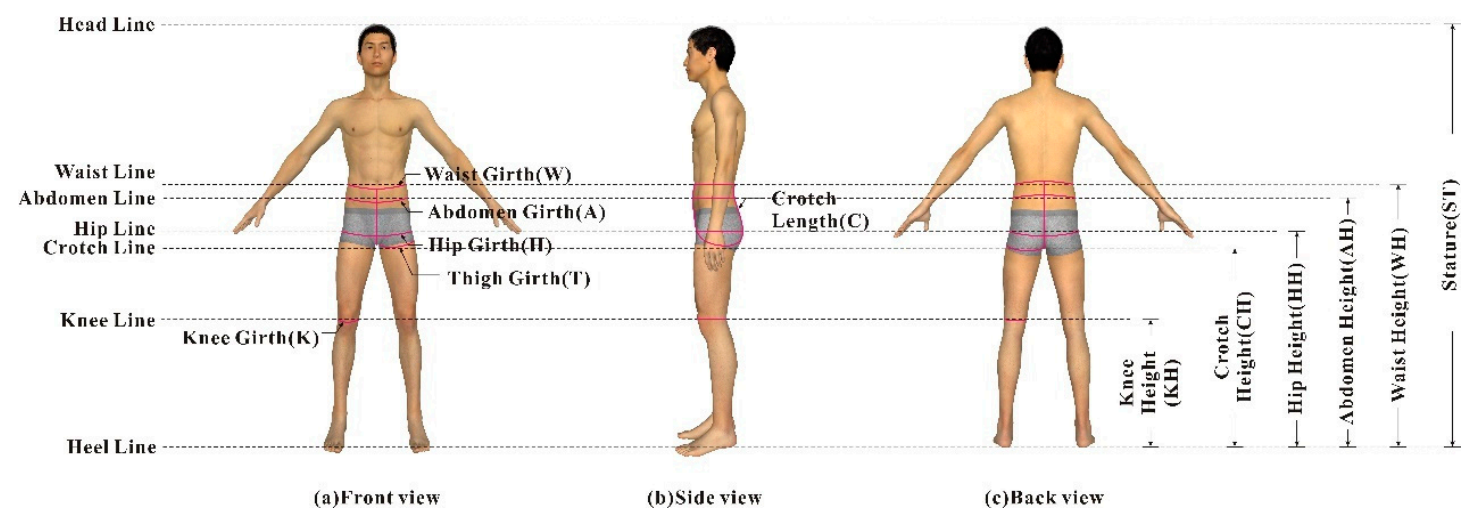

Figure 2. Diagram of the lower-body dimensions.

Table 1. Operational definitions of the selected anthropometric measurements.

\begin{tabular}{cccl}
\hline No. & Measurements & Abbr. & \\
\hline 1 & Stature & ST & The vertical distance measured from head line to heel line. \\
2 & Waist Height & WH & The vertical distance measured from waist line to heel line. \\
3 & Abdomen Height & AH & The vertical distance measured from abdomen line to heel line. \\
4 & Hip Height & HH & The vertical distance measured from hip line to heel line. \\
5 & Crotch Height & CH & The vertical distance measured from crotch line to heel line. \\
6 & Knee Height & KH & The vertical distance measured from knee line to heel line. \\
7 & Waist Girth & W & The length measured around the slenderest part of the waist horizontally. \\
8 & Abdomen Girth & A & The length measured around the fullest part of the abdomen horizontally. \\
9 & Hip Girth & $\mathrm{H}$ & The length measured around the fullest part of the hip horizontally. \\
10 & Crotch Length & $\mathrm{C}$ & The length measured from the center point of front waist line to the \\
11 & Thigh Girth & $\mathrm{T}$ & center point of back waist line through the crotch. \\
12 & Knee Girth & $\mathrm{K}$ & The length measured around the root of the thigh horizontally. \\
\end{tabular}


Table 2. Description of the variables used to construct the radial basis function (RBF) artificial neural network (ANN) model.

\begin{tabular}{cccc}
\hline Measurements & Sample Numbers & Mean Value & Standard Deviation \\
\hline Stature $(\mathrm{cm})$ & 200 & 166.04 & 5.32 \\
Waist Height $(\mathrm{cm})$ & 200 & 102.89 & 4.31 \\
Abdomen Height $(\mathrm{cm})$ & 200 & 92.32 & 4.46 \\
Hip Height $(\mathrm{cm})$ & 200 & 80.15 & 3.85 \\
Crotch Height $(\mathrm{cm})$ & 200 & 74.62 & 4.10 \\
Knee Height $(\mathrm{cm})$ & 200 & 44.42 & 2.35 \\
Waist Girth $(\mathrm{cm})$ & 200 & 79.82 & 8.87 \\
Abdomen Girth $(\mathrm{cm})$ & 200 & 82.42 & 7.47 \\
Hip Girth $(\mathrm{cm})$ & 200 & 90.65 & 5.08 \\
Crotch Length $(\mathrm{cm})$ & 200 & 77.40 & 4.73 \\
Thigh Girth $(\mathrm{cm})$ & 200 & 51.62 & 3.98 \\
Knee Girth $(\mathrm{cm})$ & 200 & 36.18 & 2.08 \\
\hline
\end{tabular}

\subsection{Construction of the RBF ANN Model for Estimating Body Dimensions}

\subsubsection{Inputs and Outputs of the RBF ANN Model}

Figure 3 illustrates the flat patterns of active leggings with key structure lines and measurements. Compared with the anthropometric measurements shown in Figure 2 and Table 1, the correspondence between the key measurements of flat patterns and body dimensions is shown in Table 3. Therefore, four body dimensions were selected for the input neurons of the model, which were waist girth $(W)$, abdomen girth (A), hip girth (H), and stature (ST).

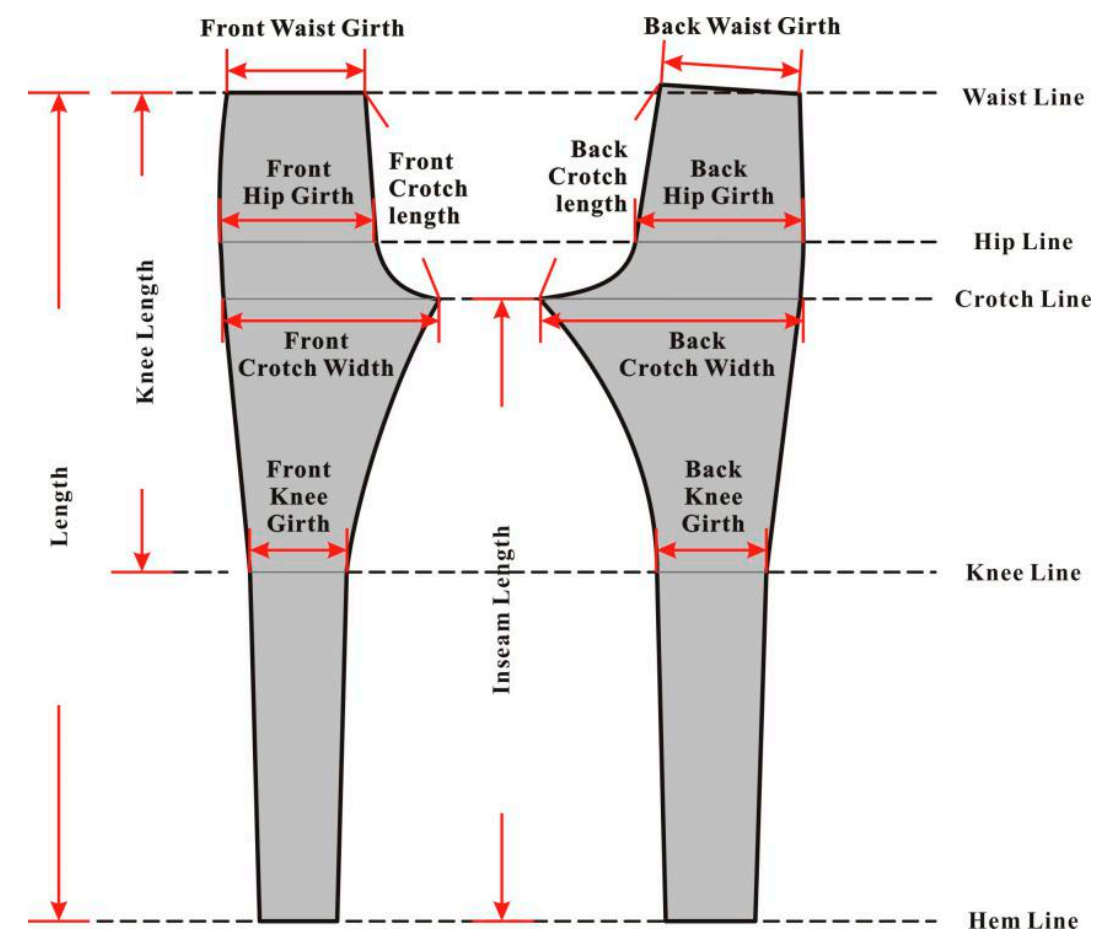

Figure 3. Flat patterns of active leggings.

The main objective of this paper was to construct an estimating model for pattern making. For the model, the inputs were easy-to-measure body dimensions, and the outputs were detailed body dimensions. As a kind of tight-fitted garment, the patterns of active leggings studied in this paper were without any ease allowance and the body dimensions outputted by the models could be utilized as the pattern dimensions. Thus, eight body dimensions were chosen for the output neurons of the 
models, including waist height (WH), abdominal height (AH), hip height $(\mathrm{HH})$, crotch height $(\mathrm{CH})$, knee height $(\mathrm{KH})$, crotch length $(\mathrm{C})$, thigh girth $(\mathrm{T})$, and knee girth $(\mathrm{K})$.

Table 3. Correspondence between the key measurements of flat patterns and body dimensions.

\begin{tabular}{|c|c|c|c|}
\hline \multirow{2}{*}{ No } & \multicolumn{2}{|c|}{ Key Measurements of Flat Patterns } & \multirow{2}{*}{ Body Dimensions } \\
\hline & Front & Back & \\
\hline 1 & Front Waist Girth & Back Waist Girth & $\mathrm{W}$ \\
\hline 2 & Front Hip Girth & Back Waist Girth & $\mathrm{H}$ \\
\hline 3 & Front Crotch Width & Back Crotch Width & $\mathrm{T}$ \\
\hline 4 & Front Knee Girth & Back Knee Girth & K \\
\hline 5 & \multicolumn{2}{|c|}{ Length } & WH \\
\hline 6 & \multicolumn{2}{|c|}{ Knee Length } & $\mathrm{WH}, \mathrm{KH}$ \\
\hline 7 & \multicolumn{2}{|c|}{ Inseam Length } & $\mathrm{CH}$ \\
\hline
\end{tabular}

The architecture of the predictive model proposed in this study is shown in Figure 4. The model based on the RBF ANN had three layers, which were one input layer with four neurons, one output layer with eight neurons, and one hidden layer. The four neurons in the input layer were the easy-to-measure key dimensions, such as waist girth, abdomen girth, hip girth, and stature. The eight neurons in the output layer were the detailed body dimensions, including waist height, abdomen height, hip height, crotch height, knee height, crotch length, thigh girth, and knee girth.

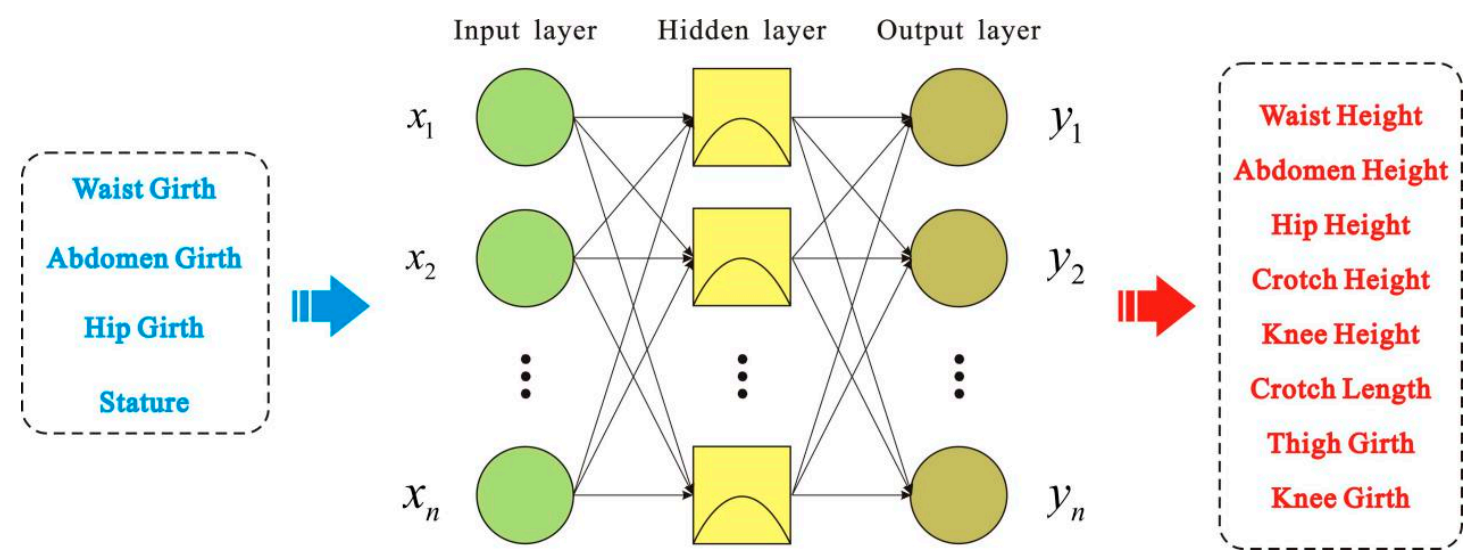

Figure 4. Architecture of the radial basis function (RBF) artificial neural networks (ANN) for estimating body dimensions.

\subsubsection{Training Dataset and Testing Dataset}

Table 4 shows the dataset used in this study, including training dataset and testing dataset. Since the sample size was too large, only part of the data are shown in Table 4.

Table 4. Parts of the data used to construct RBF ANN model (unit: $\mathrm{cm}$ ).

\begin{tabular}{|c|c|c|c|c|c|c|c|c|c|c|c|c|}
\hline \multirow{2}{*}{ SN } & \multicolumn{4}{|c|}{ Input Data } & \multicolumn{8}{|c|}{ Output Data } \\
\hline & $\mathbf{W}$ & A & $\mathbf{H}$ & ST & WH & $\mathbf{A H}$ & $\mathbf{H H}$ & $\mathrm{CH}$ & $\mathbf{K H}$ & $\mathrm{C}$ & $\mathbf{T}$ & $\mathbf{K}$ \\
\hline 1 & 85.2 & 85.3 & 89.8 & 157.0 & 97.5 & 89.4 & 78.4 & 71.1 & 42.5 & 72.3 & 52.5 & 35.2 \\
\hline 2 & 75.0 & 83.2 & 86.4 & 161.1 & 101.6 & 90.4 & 78.2 & 72.3 & 43.1 & 75.8 & 46.8 & 34.9 \\
\hline 3 & 80.9 & 81.0 & 90.3 & 164.9 & 99.3 & 92.7 & 78.6 & 72.1 & 42.1 & 75.5 & 50.5 & 37.0 \\
\hline 4 & 88.0 & 89.8 & 97.4 & 169.5 & 106.8 & 94.0 & 79.8 & 75.6 & 41.9 & 81.9 & 57.2 & 38.8 \\
\hline 5 & 79.7 & 78.5 & 90.7 & 163.9 & 101.4 & 91.3 & 78.4 & 73.7 & 44.4 & 74.8 & 51.7 & 35.9 \\
\hline 6 & 73.9 & 75.7 & 87.2 & 161.7 & 100.4 & 91.7 & 76.8 & 71.0 & 42.7 & 75.5 & 51.9 & 35.3 \\
\hline 7 & 89.0 & 87.8 & 99.3 & 174.0 & 108.6 & 99.3 & 89.0 & 82.2 & 50.1 & 74.0 & 56.2 & 41.4 \\
\hline
\end{tabular}


Table 4. Cont.

\begin{tabular}{|c|c|c|c|c|c|c|c|c|c|c|c|c|}
\hline \multirow{2}{*}{ SN } & \multicolumn{4}{|c|}{ Input Data } & \multicolumn{8}{|c|}{ Output Data } \\
\hline & $\mathbf{W}$ & $\mathbf{A}$ & $\mathbf{H}$ & ST & WH & $\mathbf{A H}$ & HH & $\mathrm{CH}$ & KH & C & $\mathbf{T}$ & $\mathbf{K}$ \\
\hline 8 & 82.7 & 80.9 & 90.3 & 169.4 & 100.7 & 91.4 & 78.7 & 71.0 & 45.9 & 74.5 & 54.3 & 36.6 \\
\hline 9 & 74.5 & 86.3 & 91.9 & 176.0 & 109.4 & 85.0 & 84.2 & 79.3 & 51.3 & 79.6 & 50.3 & 36.6 \\
\hline 10 & 80.6 & 84.3 & 90.3 & 171.1 & 105.5 & 97.0 & 82.2 & 77.9 & 47.1 & 79.2 & 51.4 & 36.4 \\
\hline 11 & 88.5 & 87.6 & 91.6 & 163.6 & 101.3 & 89.8 & 80.3 & 73.6 & 45.6 & 75.7 & 51.3 & 36.4 \\
\hline 12 & 92.1 & 95.4 & 95.4 & 166.0 & 104.3 & 96.8 & 78.8 & 73.3 & 42.7 & 84.0 & 53.4 & 37.7 \\
\hline 13 & 77.9 & 78.6 & 85.2 & 165.2 & 104.5 & 97.2 & 76.5 & 73.4 & 44.0 & 79.0 & 46.4 & 35.2 \\
\hline 14 & 87.1 & 87.6 & 91.6 & 166.3 & 100.8 & 90.3 & 80.0 & 70.3 & 43.3 & 80.9 & 52.8 & 37.5 \\
\hline 15 & 75.5 & 81.0 & 92.4 & 173.1 & 108.9 & 98.4 & 83.6 & 79.5 & 48.0 & 79.7 & 51.2 & 37.3 \\
\hline 16 & 80.3 & 87.3 & 94.5 & 175.6 & 108.3 & 98.0 & 83.3 & 79.2 & 50.4 & 80.2 & 55.4 & 37.2 \\
\hline 17 & 71.0 & 72.8 & 83.8 & 156.3 & 97.0 & 80.3 & 74.0 & 70.0 & 42.0 & 71.5 & 50.9 & 37.1 \\
\hline 18 & 74.7 & 82.3 & 87.2 & 159.0 & 96.4 & 84.3 & 74.5 & 69.4 & 41.5 & 73.9 & 49.8 & 34.4 \\
\hline 19 & 92.8 & 97.3 & 96.0 & 164.1 & 100.2 & 86.9 & 78.3 & 71.5 & 42.3 & 82.4 & 56.6 & 38.2 \\
\hline 20 & 82.9 & 83.5 & 90.9 & 183.2 & 116.4 & 104.4 & 92.2 & 90.0 & 53.2 & 79.9 & 54.4 & 36.2 \\
\hline
\end{tabular}

\subsection{Simulation of RBF ANN Model for Estimating Body Dimensions}

The radical basic function (RBF) ANN is one of the feedforward neural networks with simple structure and has the advantages of approximating any non-linear function with arbitrary accuracy and fast convergence $[16,18]$. Therefore, the predictive model proposed in this study was constructed based on RBF ANN with the architecture composed of an input layer, hidden layer, and output layer. In this paper, the simulation process included eight steps as follows.

Step 1: Distributed the element of $X=[x(1), x(2), \ldots, x(p)]^{T}$ to the input layer. Where, $X$ was a matrix of inputting samples; $p$ was the number of input samples.

Step 2: Calculated the inputs of the hidden layer;

$$
h i_{j}(k)=\sum_{1}^{n} w_{i j} x_{i}(k), j=1,2, \ldots, h
$$

where, $h i_{j}(k)$ was the inputs of the $k$ th inputting sample in the $j$ th node of hidden layer, $k=1,2, \ldots$, $p$; $w_{i j}$ was the linkage weights between input layer and hidden layer. For the RBF ANN, the linkage weights between the input layer and the hidden layer were set to 1 .

Step 3: Calculated the cluster centers $\left\{c_{1}, c_{2}, \ldots, c_{h}\right\}$ and the parameter $\sigma$ in the hidden layer;

For the RBF ANN, the outputs of the hidden layer were activated by a radial Gauss function $G$ :

$$
G\left(x(k), c_{i}\right)=\exp \left[-\frac{\left\|x(k)-c_{i}\right\|}{2 \sigma^{2}}\right], i=1,2, \ldots, h
$$

where, $c_{i}$ was the $i$ th cluster center in the hidden layer; $\sigma$, also known as spread, was the smoothing parameter, $\|\cdot\|$ was the representative of the Euclidean norm. Thus, the cluster centers $\left\{c_{1}, c_{2}, \ldots\right.$, $\left.c_{h}\right\}$ and the parameter $\sigma$ was set to 1.0 initially in this study. The cluster centers $\left\{c_{1}, c_{2}, \ldots, c_{h}\right\}$ were determined by the K-means algorithm. The operational procedures were as follows:

(1) An initial set of cluster centers $\left\{c_{1}, c_{2}, \ldots, c_{h}\right\}$ was chosen from the inputs of hidden layer randomly;

(2) Each of the input of hidden layer was assigned to its closest cluster center according to the Euclidean metrics;

(3) New cluster centers were computed as the means of the Kth cluster;

(4) If the position of any cluster center changed, return to (2), otherwise, stop. 
Step 4: Calculated the outputs of the hidden layer;

The outputs of hidden layer were calculated based on a radial Gauss function $G$ shown as Formula (2).

$$
h o_{j}(k)=\exp \left[-\frac{\left\|x(k)-c_{j}\right\|}{2 \sigma^{2}}\right], j=1,2, \ldots, h
$$

where, $h o_{j}(k)$ was the outputs of the $k$ th inputting sample in the $j$ th node of the hidden layer.

Step 5: Calculated the inputs and outputs of the output layer;

$$
\begin{gathered}
y i_{o}(k)=\sum_{1}^{h} w_{i o} h o_{j}(k), o=1,2, \ldots, m \\
y o_{o}(k)=f\left(y i_{o}(k)\right)
\end{gathered}
$$

where, $y i_{o}(k)$ was the inputs of the $k$ th inputting sample in the oth node of the output layer; $y o_{o}(k)$ referred to the output of the $k$ th inputting sample in the oth node of the output layer; $w_{i o}$ was the linkage weights between the hidden layer and the output layer; $f(\cdot)$ was the activation function in the output layer and the Sigmoid function was used as the activation function in this study. Thus, the outputs of the output layer could be calculated:

$$
f\left(y i_{o}(k)\right)=\frac{1}{1+e^{-y i_{o}(k)}}
$$

Step 6: Updated the linkage weights between the hidden layer and the output layer.

The linkage weights between the hidden layer and the output layer were amended according to Formulas (8) and (9).

$$
\begin{gathered}
w_{i o}^{N+1}=\alpha w_{i o}^{N}+\Delta w_{i o}^{N} \\
\Delta w_{i o}=-\eta \frac{\partial E}{\partial w_{i o}}
\end{gathered}
$$

where, $\alpha$ referred to the momentum factor, $\alpha$ and $\eta$ referred to the learning speed, and both of them could accelerate the convergence rate of RBF ANN. The initial value of parameter $\alpha$ was set to 0.9 and $\eta$ was adaptive in this study.

Step 7: Calculated global error $E_{G}$.

$$
E_{G}=\sum_{1}^{p} E_{i}
$$

where, $\mathrm{E}=\frac{1}{2} \sum_{1}^{m}\left[d_{o}(k)-y_{o}(k)\right]^{2}$ was the cost function to measure errors.

Step 8: Checked whether the error of the model met the goals.

If either the error was acceptably small or other terminating conditions occurred, the model stopped. Else, return to step 2 for the next round of learning until the goals were reached.

\section{Results and Discussion}

In order to reveal the effects of the factors affecting the estimating performance of the models, including volume of training dataset, quantity of hidden neurons, parameter $\sigma$ in the hidden layer, and momentum factor $\alpha$, a series of experiments were conducted. Various models were tested by the same dataset randomly extracted from the dataset illustrated in Table 4. The testing dataset in this study in shown in Table 5 . 
Table 5. Testing dataset (unit: $\mathrm{cm}$ ).

\begin{tabular}{|c|c|c|c|c|c|c|c|c|c|c|c|c|}
\hline \multirow{2}{*}{ SN } & \multicolumn{4}{|c|}{ Input Data } & \multicolumn{8}{|c|}{ Expected Output Data } \\
\hline & $\mathbf{W}$ & A & $\mathbf{H}$ & ST & WH & AH & HH & $\mathrm{CH}$ & KH & $\mathrm{C}$ & $\mathbf{T}$ & $\mathbf{K}$ \\
\hline 1 & 101.9 & 101.9 & 102.5 & 164.0 & 103.1 & 90.3 & 80.7 & 74.3 & 43.2 & 84.0 & 58.9 & 36.8 \\
\hline 2 & 69.2 & 74.7 & 91.1 & 174.6 & 108.4 & 97.7 & 83.7 & 82.0 & 46.4 & 74.1 & 51.2 & 35.7 \\
\hline 3 & 85.5 & 86.0 & 93.4 & 159.6 & 101.2 & 90.8 & 76.9 & 70.3 & 42.7 & 84.6 & 55.1 & 35.4 \\
\hline 4 & 85.5 & 90.1 & 95.9 & 158.3 & 96.6 & 83.7 & 74.1 & 67.9 & 40.1 & 77.0 & 55.0 & 34.0 \\
\hline 5 & 64.3 & 70.1 & 80.6 & 163.3 & 101.1 & 90.3 & 77.5 & 75.2 & 42.5 & 73.4 & 43.7 & 33.1 \\
\hline 6 & 71.6 & 76.1 & 86.9 & 161.3 & 97.3 & 85.8 & 76.3 & 71.6 & 41.2 & 71.9 & 50.7 & 34.7 \\
\hline 7 & 64.3 & 72.5 & 79.3 & 164.3 & 101.8 & 92.6 & 79.2 & 75.6 & 43.9 & 69.2 & 42.9 & 33.1 \\
\hline 8 & 72.5 & 77.5 & 87.7 & 171.0 & 106.9 & 94.3 & 81.1 & 77.9 & 46.6 & 72.0 & 48.4 & 34.1 \\
\hline 9 & 93.9 & 95.4 & 94.5 & 160.7 & 101.9 & 89.6 & 80.5 & 75.3 & 43.6 & 74.5 & 52.0 & 37.2 \\
\hline 10 & 96.0 & 94.7 & 100.3 & 168.8 & 104.3 & 92.5 & 81.6 & 77.4 & 44.8 & 80.0 & 57.0 & 38.4 \\
\hline 11 & 86.1 & 89.2 & 95.9 & 166.2 & 100.4 & 88.6 & 74.6 & 69.7 & 41.1 & 85.3 & 55.9 & 38.6 \\
\hline 12 & 72.4 & 76.4 & 90.9 & 173.2 & 108.7 & 99.5 & 85.7 & 80.6 & 47.3 & 72.2 & 49.7 & 35.0 \\
\hline 13 & 80.0 & 80.5 & 87.9 & 167.3 & 106.3 & 96.2 & 84.0 & 78.5 & 44.5 & 65.5 & 50.4 & 35.2 \\
\hline 14 & 72.9 & 78.3 & 89.1 & 174.7 & 97.0 & 83.8 & 83.0 & 79.7 & 46.8 & 76.0 & 49.2 & 37.0 \\
\hline 15 & 78.4 & 78.9 & 88.1 & 167.5 & 101.3 & 92.1 & 81.6 & 76.8 & 44.3 & 71.9 & 52.3 & 36.2 \\
\hline 16 & 68.0 & 71.0 & 82.4 & 168.3 & 103.6 & 96.1 & 82.6 & 77.5 & 45.3 & 65.8 & 45.6 & 32.8 \\
\hline 17 & 75.8 & 81.3 & 95.6 & 172.7 & 107.7 & 98.3 & 86.3 & 81.4 & 47.0 & 73.8 & 55.8 & 35.6 \\
\hline 18 & 74.7 & 81.0 & 92.2 & 171.2 & 109.0 & 93.2 & 81.7 & 76.7 & 45.1 & 81.8 & 53.8 & 37.4 \\
\hline 19 & 61.4 & 63.2 & 79.2 & 165.6 & 101.9 & 92.0 & 79.6 & 76.9 & 44.5 & 70.0 & 43.7 & 31.8 \\
\hline 20 & 71.2 & 76.5 & 89.5 & 169.7 & 103.3 & 92.5 & 81.3 & 75.4 & 44.5 & 76.2 & 52.2 & 36.0 \\
\hline
\end{tabular}

Note: $\mathrm{SN}$ is testing sample number. For W, A, H, ST, WH, AH, HH, CH, C, T, K, please refer to Figure 2 and Table 1.

After the model was tested, $d_{i j}$ were calculated according to Formula (10), which was defined as the absolute deviation between $Y_{i j}$ and $Y^{\prime}{ }_{i j}$. $D$ was defined as a matrix composed of $d_{i j}$ according to formula (11). MSED referred to mean square error of $D$, which was utilized to evaluate the predictive precision of the models.

$$
\begin{gathered}
d_{i j}=Y_{i j}-Y_{i j}^{\prime}, i=1,2, \ldots, 20, j=1,2, \ldots, 8 \\
D=\left(\begin{array}{ccc}
d_{11} & \cdots & d_{1 j} \\
\vdots & \ddots & \vdots \\
d_{i 1} & \cdots & d_{i j}
\end{array}\right), i=1,2, \ldots, 20, j=1,2, \ldots, 8
\end{gathered}
$$

where, $Y_{i j}$ and $Y_{i j}^{\prime}$ were respectively defined as the estimated output data and the expected output data of the $i$ th inputting sample in the $j$ th node of output layer.

\subsection{Effects of Volume of Training Dataset}

Since the training dataset was one of the key factors affecting the performance of the RBF ANN model, five various training datasets with 100, 120, 140, 160, and 180 samples, respectively, were established in order to investigate the effects. For each training dataset, the data were extracted randomly from the remaining data in the dataset shown in Table 4. Then, an RBF ANN model with 4 input neurons, 20 hidden neurons, and 8 output neurons were trained by the five sets, respectively, using the same parameter $\sigma(\sigma=1.0)$ in the hidden layer and momentum factor $\alpha(\alpha=0.9)$. After being well-trained, the five models were tested by the datasets shown in Table 5 .

Figure 5 illustrates the MSED of the five models. It can be easily seen that the MSE dropped as the volume of training datasets increased from 100 to 180. Among the five models, the MSED of the model trained by 180 samples was the lowest. From the perspective of MSED, the precision of the models grew with the increase in training samples. It means that the more trained data the models learned, the stronger were their estimating ability. 


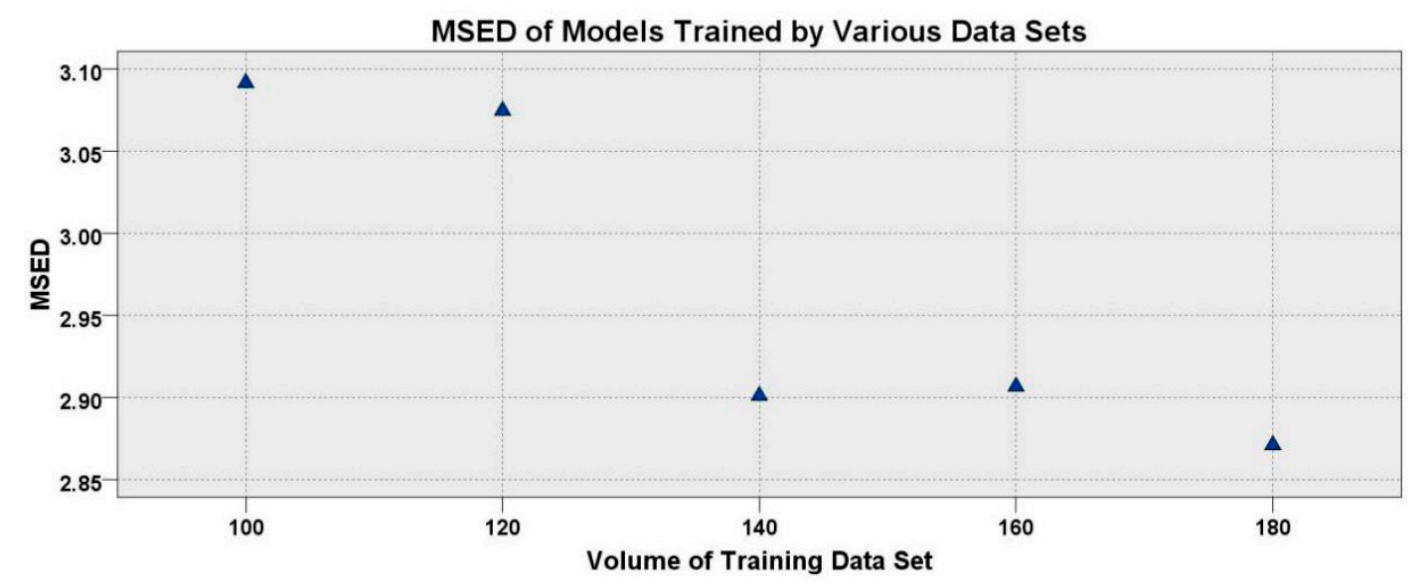

Figure 5. Mean square error of $D$ (MSED) of the RBF ANN models trained by datasets with various volumes.

\subsection{Effects of the Quantity of Hidden Neurons}

Quantity of neurons in the hidden layer had great impact on the performance of the RBF ANN. Generally, the precision of the RBF ANN could be improved as the quantity of the hidden layer increased. However, too many neurons in the hidden layer will prolong the convergence rate of the RBF ANN, and even lead to failure to train the model. In order to determine the optimum number of hidden neurons, multiple experiments were executed. Twelve RBF ANN models with various hidden layer were constructed firstly. The quantity of hidden neurons were $60,65,70,71,72,73,75,80,85,90$, 95, and 100, respectively. The parameter $\sigma$ and $\alpha$ were still set to 1.0 and 0.9 . Afterwards, the twelve models were trained by the same dataset with 180 samples. Finally, they were tested with the dataset in Table 5.

The MSED of the models with various hidden layers are shown in Table 6. When the quantity of the hidden neurons increased from 60 to 72, the MSED declined gradually, while rising gradually as the quantity of the hidden neurons increased from 72 to 90 . From 90 to 100, the MSED descended again. Overall, the MSED of the model with 72 hidden neurons has the minimum value relatively. Therefore, it could be considered that the precision of the RBF ANN model reached its maximum when the quantity of neurons in the hidden layer was 72 .

Table 6. Mean square error of $D$ (MSED) of models with various hidden layers.

\begin{tabular}{ccc}
\hline Model No. & Number of Hidden Neurons & MSED \\
\hline 1 & 60 & 2.8904 \\
2 & 65 & 2.8521 \\
3 & 70 & 2.8351 \\
4 & 71 & 2.8528 \\
5 & 72 & 2.8055 \\
6 & 73 & 2.8284 \\
7 & 75 & 2.8306 \\
8 & 80 & 2.8409 \\
9 & 85 & 2.8454 \\
10 & 90 & 2.8919 \\
11 & 95 & 2.8708 \\
12 & 100 & 2.8386 \\
\hline
\end{tabular}

\subsection{Effects of the Parameter $\sigma$ in the Hidden Layer}

In the hidden layer of the RBF ANN, since the outputs were activated by Formula (2), the parameter $\sigma$ should have influenced the estimating performance. Based on the findings of Sections 3.1 and 3.2, thirteen RBF ANN models with four inputs, 72 hidden neurons, and eight outputs 
were built for further study. The only difference among them was the parameter $\sigma$, which were 0.001 , $0.005,0.008,0.01,0.011,0.012,0.013,0.015,1,1.25,1.67,2.5$, and 5 , respectively. After being well-trained and tested, the models were compared according to MSED. Table 7 shows the MSED of the thirteen models. As $\sigma$ altered from 0.001 to 5, the MSED presented a tendency to descend first and then rise. When $\sigma$ was at 0.012 , MSED came to the minimum. Thus, the optimum value of $\sigma$ was determined.

Table 7. The MSED of the models with various parameters of $\sigma$ in the hidden layer.

\begin{tabular}{ccc}
\hline Model No. & Parameter $\sigma$ & MSED \\
\hline 1 & 0.001 & 2.7423 \\
2 & 0.005 & 2.7395 \\
3 & 0.008 & 2.7293 \\
4 & 0.010 & 2.7283 \\
5 & 0.011 & 2.7280 \\
6 & 0.012 & 2.7279 \\
7 & 0.013 & 2.7284 \\
8 & 0.015 & 2.7292 \\
9 & 1.000 & 2.8055 \\
10 & 1.250 & 2.9829 \\
11 & 1.670 & 2.9254 \\
12 & 2.500 & 3.0759 \\
13 & 5.000 & 3.4778 \\
\hline
\end{tabular}

\subsection{Effects of the Momentum Factor a}

In order to find how the momentum factor affected the RBF ANN model in this study, ten kinds of $\alpha$ were selected between 0.1 and 1 , and they were $0.1,0.2,0.3,0.4,0.5,0.6,0.7,0.8,0.9$, and 1 . The RBF ANN model constructed based on the findings of Section 3.3 were trained ten times according to the $\alpha$ selected. Although the $\alpha$ was changing, MSED was almost invariant. The running time of the model expended gradually with the increase of $\alpha$.

\subsection{Comparison with Linear Regression Model and BP ANN Model}

Table 8 shows the MSED of three predictive models, which were the RBF ANN model with the optimum performance constructed in this study, the BP ANN model, and the linear regression model. For each anthropometric measurement, the MSED of RBF ANN model was less than that of the other two models, which meant that the estimating performance of the RBF ANN model was better than the others.

Table 8. The MSED of various predictive models.

\begin{tabular}{cccc}
\hline \multirow{2}{*}{ Measurements } & \multicolumn{3}{c}{ MSED } \\
\cline { 2 - 4 } & RBF ANN Model & BP ANN Model & Linear Regression Model \\
\hline Waist Height & 3.0814 & 3.1185 & 3.1854 \\
Abdomen Height & 3.0197 & 3.7809 & 3.7631 \\
Hip Height & 1.8514 & 2.0029 & 2.0887 \\
Crotch Height & 1.0450 & 1.1139 & 2.0705 \\
Knee Height & 1.9307 & 2.0918 & 2.0084 \\
Crotch Length & 3.9251 & 4.0531 & 5.9871 \\
Thigh Girth & 1.4631 & 1.7397 & 2.5929 \\
Knee Girth & 1.0238 & 1.1137 & 1.4043 \\
\hline
\end{tabular}

\subsection{Application of the Body Dimensions Estimated by RBF ANN Model}

In order to verify the model presented in this study, tight-fitted active leggings patterns making was taken for use as a case study. Due to tight fitness, the outputted body dimensions could be used as pattern dimensions of active leggings. 
The application process in this study in illustrated in Figure 6, as follows:

Initially, anthropometric data of a subject were selected randomly. The subject's stature was $171.8 \mathrm{~cm}$, waist girth was $68.9 \mathrm{~cm}$, abdomen girth was $77.2 \mathrm{~cm}$, and hip girth was $88.9 \mathrm{~cm}$. Therefore, the input vector $x=(171.8,68.9,77.2,88.9)$. The desired output vector $d=(105.8,94.7,82.8,77.4$, $45.2,77.2,49.2,35.4)$ was composed of the real dimensions of the subject, which represented waist height, abdomen height, hip height, crotch height, knee height, crotch length, thigh girth, and knee girth, respectively.

Secondly, the RBF ANN was utilized to estimate the body dimensions related to making the patterns of active leggings. After the vector $d$ was inputted into the predictive model based on RBF ANN, the output vector $y$ was generated, as follows:

$$
y=(106,94.1,83.2,77.6,45.9,74.9,48.8,35.2)
$$

Sequentially, the body dimensions generated by the RBF ANN model were employed to making the patterns of active leggings. Then, the patterns were joined together into $3 \mathrm{D}$ active leggings by virtual stitching technology.

Finally, the stitched leggings were tried on by a virtual avatar to evaluate the fitness.

The available evidence lent support to the view that the proposed RBF ANN model could estimate the body dimensions related to active leggings pattern making efficiently and precisely. The predictive accuracy would be further improved by more training data.

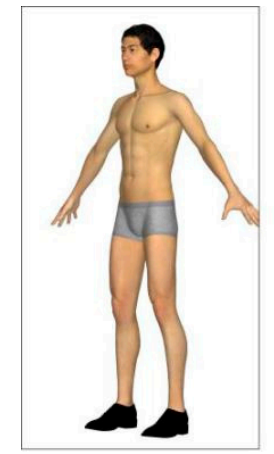

(a) Data Acquisition

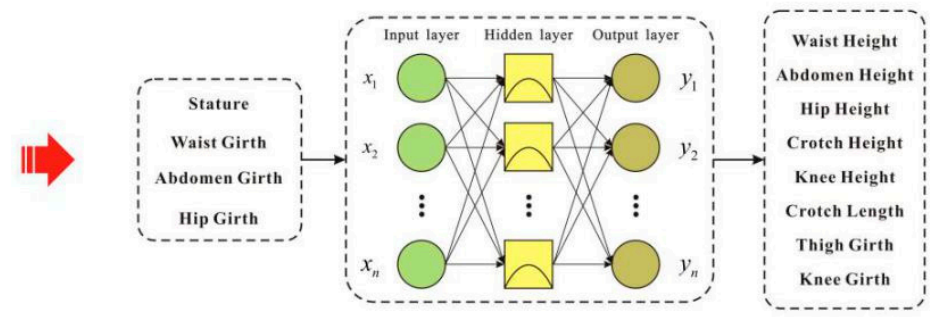

(b) Body Dimensions Generation
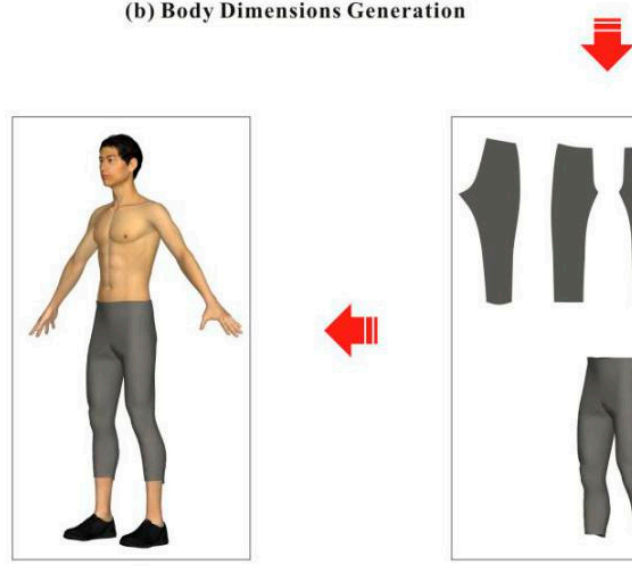

(d)Try-on Validation

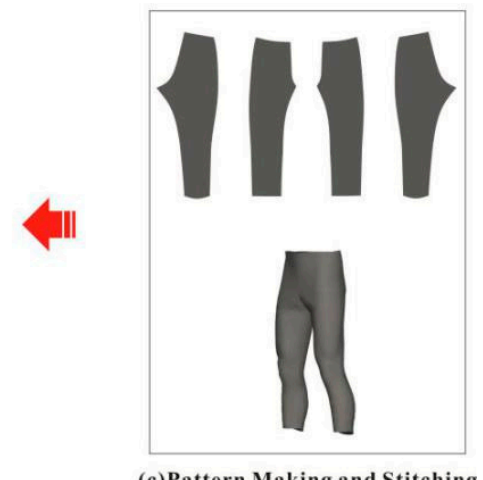

(c)Pattern Making and Stitching

Figure 6. Application of the body dimensions predictive model based on RBF ANN.

\section{Conclusions}

In order to meet the personalized requirements of consumers in the internet era, this study has proposed a new neural networks method based on RBF ANN for garment pattern making, especially for tight-fitted garments, which is less time-consuming and more accurate than current methods. The key factors affecting the models, such as volume of the training dataset, quantity of hidden neurons, spreading parameter $\sigma$, and momentum factor $\alpha$, were analyzed. After multiple experiments, 
the optimum parameters were determined. When spreading parameter $\sigma$ and momentum factor $\alpha$ were set to 0.012 and 1, the RBF ANN model with four inputs, 72 hidden neurons, and eight outputs could reach the maximum accuracy, after being trained by the dataset with 180 samples. Compared with the traditional linear regression model used in the apparel industry, the RBF ANN model was superior on predictive precision contributing to its outstanding non-linear mapping capacity. The proposed RBF ANN model had a simpler structure with higher estimating accuracy than the BP ANN model. With the approaching digital customization era in the apparel industry, it is feasible to adopt the RBF ANN model proposed in this study for a computer-aided body dimension auto-generation system, which could promote the accuracy and efficiency of pattern designers and improve the fitness of garments significantly.

Since the presented RBF ANN model in this study was trained by a dataset consisting of 200 subjects only, the accuracy of the model could be further improved. Apart from the sample capacity, the factors influencing body dimensions such as age, race, and geographical areas should be considered in future work. Although the approach proposed in this study was concentrated on making tight fitting garment patterns like active leggings, and it could also be applied in pattern dimension prediction of other garments with different fitness. In the future, through inputting more learning data from experienced pattern makers, the proposed model could be used by pattern makers lacking in related expertise and experience.

Author Contributions: Conceptualization, Z.W. and J.W.; Methodology, Z.W. and Y.X.; Funding acquisition, Z.W., Y.X. and K.L.; Supervision, J.W.; Validation, Z.W., Y.X. and Y.Y.; Writing-original draft, Z.W.

Funding: This research was funded by the key Research Project of Humanities and Social Sciences in Anhui Province College (grant number SK2016A0116 and SK2017A0119), the Open Project Program of Anhui Province College of Anhui Province College Key Laboratory of Textile Fabrics, Anhui Engineering and Technology Research Center of Textile (grant number 2018AKLTF15), the Humanities and Social Sciences Research Project of higher Education Promotion Program in Anhui Province College (grant number TSSK2016B20), the National Natural Science Foundation of China (grant number 61806161) and Special Scientific Research Plan Projects of Shaanxi Education Department (grant number 18JK0352).

Conflicts of Interest: The authors declare no conflict of interest.

\section{References}

1. Daanen, H.M.; Van De Water, G.J. Whole body scanners. Displays 1998, 19, 111-120. [CrossRef]

2. Daanen, H.A.M.; Ter Haar, F.B. 3D whole body scanners revisited. Displays 2013, 34, 270-275. [CrossRef]

3. Uhm, T.; Park, H.; Park, J.-I. Fully vision-based automatic human body measurement system for apparel application. Measurement 2015, 61, 169-179. [CrossRef]

4. Wu, G.; Liu, S.; Wu, X.; Ding, X. Research on lower body shape of late pregnant women in Shanghai area of China. Int. J. Ind. Ergon. 2015, 46, 69-75. [CrossRef]

5. Lacko, D.; Huysmans, T.; Vleugels, J.; de Bruyne, G.; van Hulle, M.M.; Sijbers, J.; Verwulgen, S. Product sizing with 3D anthropometry and k-medoids clustering. Comput.-Aided Des. 2017, 91, 60-74. [CrossRef]

6. Agha, S.R.; Alnahhal, M.J. Neural network and multiple linear regression to predict school children dimensions for ergonomic school furniture design. Appl. Ergon. 2012, 43, 979-984. [CrossRef] [PubMed]

7. Chan, A.P.; Fan, J.; Yu, W.M. Prediction of men's shirt pattern based on 3D body measurements. Int. J. Cloth. Sci. Technol. 2005, 17, 100-108. [CrossRef]

8. Ngassa, C.N.; Akanbi, O.G.; Ismaila, S.O. Models for estimating the anthropometric dimensions using standing height for furniture design. Eur. J. Mark. 2014, 12, 336-347.

9. Poirson, E.; Parkinson, M. Estimated anthropometry for male commercial pilots in Europe and an approach to its use in seat design. Int. J. Ind. Ergon. 2014, 44, 769-776. [CrossRef]

10. Zhao, N.; Li, Z. Viscosity Prediction of Different Ethylene Glycol/Water Based Nanofluids Using a RBF Neural Network. Appl. Sci. 2017, 7, 409. [CrossRef]

11. Almeida, A.; Azkune, G. Predicting Human Behaviour with Recurrent Neural Networks. Appl. Sci. 2018, 8, 305. [CrossRef]

12. Liu, Y.; Zhao, J.; Xiao, Y. C-RBFNN: A user retweet behavior prediction method for hotspot topics based on improved RBF neural network. Neurocomputing 2018, 275, 733-746. [CrossRef] 
13. Shi, X.; Cheng, Y.; Yin, C.; Huang, X.; Zhong, S. Design of adaptive backstepping dynamic surface control method with RBF neural network for uncertain nonlinear system. Neurocomputing 2018, 330, 490-503. [CrossRef]

14. Zhang, X.; Sun, S.; Li, C.; Tang, Z. Impact of Load Variation on the Accuracy of Gait Recognition from Surface EMG Signals. Appl. Sci. 2018, 8, 1462. [CrossRef]

15. Chan, A.P.; Fan, J.; Yu, W. Men's Shirt Pattern Design Part II: Prediction of Pattern Parameters from 3D Body Measurements. FIBER 2003, 59, 328-333. [CrossRef]

16. Liu, Z.; Li, J.; Chen, G.; Lu, G. Predicting detailed body sizes by feature parameters. Int. J. Cloth. Sci. Technol. 2014, 26, 118-130. [CrossRef]

17. Liu, K.; Wang, J.; Kamalha, E.; Li, V.; Zeng, X. Construction of a prediction model for body dimensions used in garment pattern making based on anthropometric data learning. J. Text. Inst. 2017, 108, 2107-2114. [CrossRef]

18. Meyer-Baese, A.; Schmid, V. Chapter 7-Foundations of Neural Networks. In Pattern Recognition and Signal Analysis in Medical Imaging, 2nd ed.; Meyer-Baese, A., Schmid, V., Eds.; Academic Press: Oxford, UK, 2014; pp. 197-243.

(C) 2019 by the authors. Licensee MDPI, Basel, Switzerland. This article is an open access article distributed under the terms and conditions of the Creative Commons Attribution (CC BY) license (http:/ / creativecommons.org/licenses/by/4.0/). 